\title{
Target mass corrections for the virtual photon structure functions to the next-to-next-to-leading order in QCD
}

\author{
Yoshio Kitadono* \\ Department of Physics, Faculty of Science, Hiroshima University, Higashi Hiroshima 739-8526, Japan \\ Ken Sasaki ${ }^{\dagger}$ \\ Department of Physics, Faculty of Engineering, Yokohama National University, Yokohama 240-8501, Japan \\ Takahiro Ueda ${ }^{*}$ \\ High Energy Accelerator Research Organization (KEK), 1-1 Oho, Tsukuba, Ibaraki 305-0801, Japan \\ Tsuneo Uematsu ${ }^{\S}$ \\ Department of Physics, Graduate School of Science, Kyoto University, Yoshida, Kyoto 606-8501, Japan
}

(Received 8 January 2008; published 24 March 2008)

We investigate target mass effects in the unpolarized virtual photon structure functions $F_{2}^{\gamma}\left(x, Q^{2}, P^{2}\right)$ and $F_{L}^{\gamma}\left(x, Q^{2}, P^{2}\right)$ in perturbative QCD for the kinematical region $\Lambda^{2} \ll P^{2} \ll Q^{2}$, where $-Q^{2}\left(-P^{2}\right)$ is the mass squared of the probe (target) photon and $\Lambda$ is the QCD scale parameter. We obtain the Nachtmann moments for the structure functions and then, by inverting the moments, we get the expressions in closed form for $F_{2}^{\gamma}\left(x, Q^{2}, P^{2}\right)$ up to the next-to-next-to-leading order and for $F_{L}^{\gamma}\left(x, Q^{2}, P^{2}\right)$ up to the next-to-leading order, both of which include the target mass corrections. Numerical analysis exhibits that target mass effects appear at large $x$ and become sizable near $x_{\max }(=$ $\left.1 /\left(1+\frac{P^{2}}{Q^{2}}\right)\right)$, the maximal value of $x$, as the ratio $P^{2} / Q^{2}$ increases.

DOI: 10.1103/PhysRevD.77.054019

PACS numbers: 12.38.Bx, 13.60.Hb, 14.70.Bh

\section{INTRODUCTION}

It is well known that, in $e^{+} e^{-}$collision experiments, the cross section for the two-photon processes $e^{+} e^{-} \rightarrow$ $e^{+} e^{-}+$hadrons illustrated in Fig. 1 dominates at high energies over other processes such as one-photon annihilation process $e^{+} e^{-} \rightarrow \gamma^{*} \rightarrow$ hadrons. Here we consider the two-photon processes in the double-tag events, where both the outgoing $e^{+}$and $e^{-}$are detected. Especially, we investigate the case in which one of the virtual photon is far off-shell (large $Q^{2} \equiv-q^{2}$ ), while the other is close to the mass-shell (small $P^{2}=-p^{2}$ ). This process can be viewed as a deep-inelastic scattering off a photon target [1] with mass squared $-P^{2}$, through which we can study the photon structure functions.

In the case of a real photon target $\left(P^{2}=0\right)$, unpolarized (spin-averaged) photon structure functions $F_{2}^{\gamma}\left(x, Q^{2}\right)$ and $F_{L}^{\gamma}\left(x, Q^{2}\right)$ were studied first in the parton model [2], and then investigated in perturbative QCD (pQCD). In the framework based on the operator product expansion (OPE) [3] supplemented by the renormalization (RG) group method, Witten [4] obtained the leading order (LO) QCD contributions to $F_{2}^{\gamma}$ and $F_{L}^{\gamma}$ and, shortly after, the next-to-leading order (NLO) QCD corrections to $F_{2}^{\gamma}$ were calculated by Bardeen and Buras [5]. The same

\footnotetext{
*kitadono@scphys.kyoto-u.ac.jp

${ }^{\dagger}$ sasaki@phys.ynu.ac.jp

\#uedat@ post.kek.jp

§uematsu@scphys.kyoto-u.ac.jp
}

results were rederived by the QCD improved parton model approach [6,7]. The QCD analysis of the polarized photon structure function $g_{1}^{\gamma}\left(x, Q^{2}\right)$ for the real photon target was performed in the LO [8] and in the NLO $[9,10]$.

The structure functions $F_{2}^{\gamma}\left(x, Q^{2}, P^{2}\right)$ and $F_{L}^{\gamma}\left(x, Q^{2}, P^{2}\right)$ for the case of a virtual photon target $\left(P^{2} \neq 0\right)$ were studied in the LO [11] and in the NLO [12] by pQCD. In fact, these structure functions were analyzed in the kinematical region,

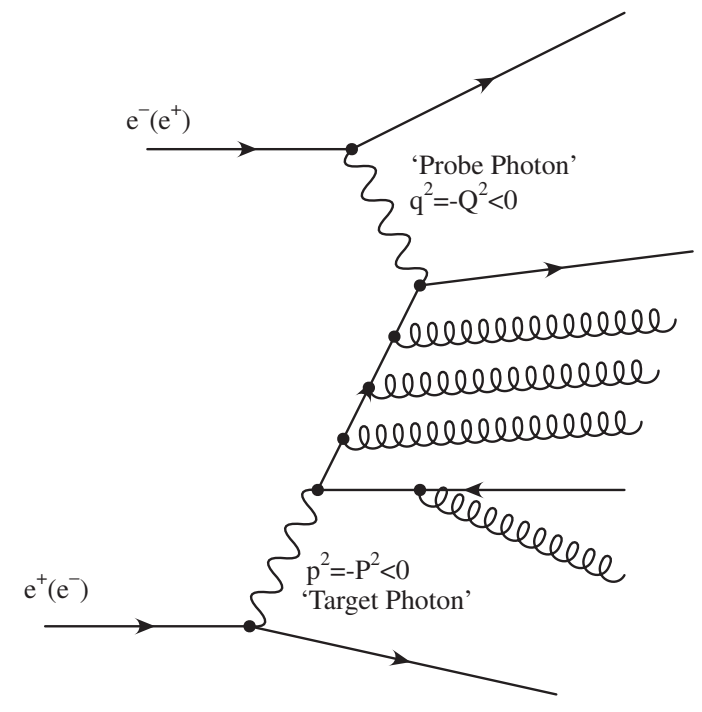

FIG. 1. Deep inelastic scattering on a virtual photon in the $e^{+} e^{-}$collider experiments. 


$$
\Lambda^{2} \ll P^{2} \ll Q^{2},
$$

where $\Lambda$ is the QCD scale parameter. The advantage of studying a virtual photon target in the kinematical region (1.1) is that we can calculate the whole structure function, its shape and magnitude, by the perturbative method. This is contrasted with the case of the real photon target where in the NLO there exist nonperturbative pieces. The virtual photon structure functions $F_{2}^{\gamma}$ and $F_{L}^{\gamma}$ were also studied by using the DGLAP-type QCD evolution equations [13-16]. In the same kinematical region (1.1), the polarized virtual photon structure function $g_{1}^{\gamma}\left(x, Q^{2}, P^{2}\right)$ was investigated up to the NLO in QCD in Ref. [17] and in the second paper of [10]. Moreover, the polarized parton distributions inside the virtual photon were analyzed in [18]. Recently the first moment of $g_{1}^{\gamma}\left(x, Q^{2}, P^{2}\right)$ was calculated up to the next-tonext-to-leading order (NNLO) [19]. For more information on the recent theoretical and experimental investigation of unpolarized and polarized photon structure, see the review articles [20].

In our previous paper [21], we have studied the unpolarized virtual photon structure functions, $F_{2}^{\gamma}\left(x, Q^{2}, P^{2}\right)$ up to the NNLO and $F_{L}^{\gamma}\left(x, Q^{2}, P^{2}\right)$ up to the NLO, in pQCD for the kinematical region (1.1). This investigation became possible thanks to the recent three-loop calculations of the parton-parton as well as photon-parton splitting functions [22-24]. There we have considered the logarithmic corrections arising from the QCD higher-order effects up to the NNLO, and ignored all the power corrections of the form $\left(P^{2} / Q^{2}\right)^{k}(k=1,2, \cdots)$ coming either from target mass effects or from higher-twist effects.

In fact, if the target is a real photon $\left(P^{2}=0\right)$, there is no need to consider target mass corrections. But when the target becomes off-shell, for example, $P^{2} \geq M^{2}$, where $M$ is the nucleon mass, and for relatively low values of $Q^{2}$, contributions suppressed by powers of $P^{2} / Q^{2}$ may become important. Then we need to take into account these target mass contributions just like the case of the nucleon structure functions. The consideration of target mass effects (TME) is important by another reason. For the virtual photon target, the maximal value of the Bjorken variable $x$ is not 1 but

$$
x_{\max }=\frac{1}{1+\frac{P^{2}}{Q^{2}}},
$$

due to the constraint $(p+q)^{2} \geq 0$, which is in contrast to the nucleon case where $x_{\max }=1$. The structure functions should vanish at $x=x_{\max }$. However, both the QCD NNLO result for $F_{2}^{\gamma}\left(x, Q^{2}, P^{2}\right)$ and the NLO result for $F_{L}^{\gamma}\left(x, Q^{2}, P^{2}\right)[21]$ show that the predicted graphs do not vanish but remains finite at $x=x_{\max }$. This flaw is coming from the fact that TME have not been taken into account in the analysis. The target mass corrections have been studied in the past for the cases of unpolarized [25-27] and polarized [28-32] nucleon structure functions. As for the polarized virtual photon structure functions $g_{1}^{\gamma}\left(x, Q^{2}, P^{2}\right)$ and $g_{2}^{\gamma}\left(x, Q^{2}, P^{2}\right)$, TME have been studied in Ref. [33].

In the present paper, we investigate the TME for the unpolarized virtual photon structure functions, $F_{2}^{\gamma}\left(x, Q^{2}, P^{2}\right)$ up to the NNLO and $F_{L}^{\gamma}\left(x, Q^{2}, P^{2}\right)$ up to the NLO, in pQCD. We use the framework of the OPE supplemented by the RG method. The photon matrix elements of the relevant traceless operators in the OPE are expressed by traceless tensors. These tensors contain many trace terms so that they satisfy the tracelessness conditions. The basic idea for computing the target mass corrections is to take account of these trace terms in the traceless tensors properly. There are two methods used so far for collecting all those trace terms. One, which was introduced by Nachtmann [25], is to make use of Gegenbauer polynomials to express the contractions between $q_{\mu_{1}} \cdots q_{\mu_{n}}$ and the traceless tensors [25,28-30]. This method leads to the Nachtmann moments for the operators with definite spin. The other, first used by Georgi and Politzer [26], is to write traceless tensors explicitly and then to collect trace terms and sum them up. Through the latter approach, the moments of structure functions are expressed as functions of the reduced operator matrix elements and coefficient functions with different spins. Actually both methods give equivalent results. In this paper we apply the former method to study the target mass corrections to the structure functions $F_{2}^{\gamma}$ and $F_{L}^{\gamma}$.

In the next section we discuss the framework for analyzing the TME based on the OPE. We introduce Gegenbauer polynomials to take account of the trace terms properly. In Sec. III we derive the Nachtmann moments for the structure functions using the orthogonality relations of Gegenbauer polynomials. In Sec. IV, by inverting the Nachtmann moments, we obtain the explicit expression for $F_{2}^{\gamma}\left(x, Q^{2}, P^{2}\right)$ [for $F_{L}^{\gamma}\left(x, Q^{2}, P^{2}\right)$ ] evaluated up to the NNLO (up to the NLO) with TME included. In Sec. V we perform the numerical analysis and show that target mass corrections become sizable near $x_{\max }$. The final section is devoted to the conclusion.

\section{OPERATOR PRODUCT EXPANSION}

We analyze the virtual photon structure functions $F_{2}^{\gamma}\left(x, Q^{2}, P^{2}\right)$ and $F_{L}^{\gamma}\left(x, Q^{2}, P^{2}\right)$ using the theoretical framework based on the OPE and the RG method. Unless otherwise stated, we will follow the notation of Ref. [5]. Let us consider the forward virtual photon scattering amplitude for $\gamma(q)+\gamma(p) \rightarrow \gamma(q)+\gamma(p)$ illustrated in Fig. 2,

$$
\begin{aligned}
T_{\mu \nu \rho \tau}(p, q)= & i \int d^{4} x d^{4} y d^{4} z e^{i q \cdot x} e^{i p \cdot(y-z)} \\
& \times\left\langle 0\left|T\left(J_{\mu}(x) J_{\nu}(0) J_{\rho}(y) J_{\tau}(z)\right)\right| 0\right\rangle,
\end{aligned}
$$

where $J_{\mu}$ is the electromagnetic current. Its absorptive part is related to the structure tensor $W_{\mu \nu \rho \tau}(p, q)$ for the target 


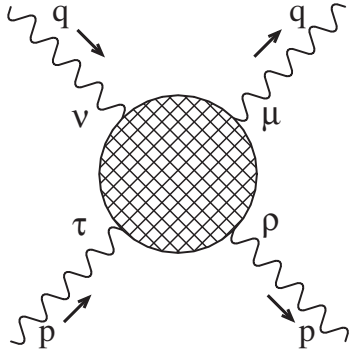

FIG. 2. Forward scattering of a virtual photon with momentum $q$ and another virtual photon with momentum $p$. The Lorentz indices are denoted by $\mu, \nu, \rho, \tau$.

photon with mass squared $p^{2}=-P^{2}$ probed by the photon with $q^{2}=-Q^{2}$ :

$$
W_{\mu \nu \rho \tau}(p, q)=\frac{1}{\pi} \operatorname{Im} T_{\mu \nu \rho \tau}(p, q) .
$$

Taking a spin average for the target photon, we get

$$
\begin{aligned}
W_{\mu \nu}^{\gamma}(p, q) & =\frac{1}{2} \sum_{\lambda} \epsilon_{(\lambda)}^{\rho *}(p) W_{\mu \nu \rho \tau}(p, q) \epsilon_{(\lambda)}^{\tau}(p) \\
& =-\frac{1}{2} g^{\rho \tau} W_{\mu \nu \rho \tau}(p, q) \\
& =\frac{1}{2} \int d^{4} x e^{i q x}\left\langle\gamma(p)\left|J_{\mu}(x) J_{\nu}(0)\right| \gamma(p)\right\rangle_{\text {spin av }} .
\end{aligned}
$$

Now $W_{\mu \nu}^{\gamma}(p, q)$ is expressed in terms of two independent structure functions $F_{L}^{\gamma}\left(x, Q^{2}, P^{2}\right)$ and $F_{2}^{\gamma}\left(x, Q^{2}, P^{2}\right)$ without neglecting the target mass squared $p^{2}$ (see Appendix A):

$$
W_{\mu \nu}^{\gamma}(p, q)=e_{\mu \nu}\left\{\frac{1}{x} F_{L}^{\gamma}+\frac{p^{2} q^{2}}{(p \cdot q)^{2}} \frac{1}{x} F_{2}^{\gamma}\right\}+d_{\mu \nu} \frac{1}{x} F_{2}^{\gamma},
$$

where

$$
\begin{gathered}
e_{\mu \nu}=g_{\mu \nu}-\frac{q_{\mu} q_{\nu}}{q^{2}}, \\
d_{\mu \nu}=-g_{\mu \nu}+\frac{p_{\mu} q_{\nu}+p_{\nu} q_{\mu}}{p \cdot q}-\frac{p_{\mu} p_{\nu}}{(p \cdot q)^{2}} q^{2},
\end{gathered}
$$

and $x$ is the Bjorken variable defined by $x=Q^{2} / 2 p \cdot q$.
Applying OPE for the product of two electromagnetic currents at short distance we get

$$
\begin{aligned}
& i \int d^{4} x e^{i q x} T\left(J_{\mu}(x) J_{\nu}(0)\right) \\
& =\left(g_{\mu \nu}-\frac{q_{\mu} q_{\nu}}{q^{2}}\right) \sum_{\substack{n=0 \\
n=\text { even }}}\left(\frac{2}{Q^{2}}\right)^{n} q_{\mu_{1}} \cdots q_{\mu_{n}} \sum_{i} C_{L, n}^{i} O_{i}^{\mu_{1} \cdots \mu_{n}} \\
& \quad+\left(-g_{\mu \lambda} g_{\nu \sigma} q^{2}+g_{\mu \lambda} q_{\nu} q_{\sigma}+g_{\nu \sigma} q_{\mu} q_{\lambda}\right. \\
& \left.\quad-g_{\mu \nu} q_{\lambda} q_{\sigma}\right) \sum_{\substack{n=2 \\
n=\text { even }}}\left(\frac{2}{Q^{2}}\right)^{n} q_{\mu_{1}} \cdots q_{\mu_{n-2}} \sum_{i} C_{2, n}^{i} O_{i}^{\lambda \sigma \mu_{1} \cdots \mu_{n-2}} \\
& \quad+\cdots,
\end{aligned}
$$

where $C_{L, n}^{i}$ and $C_{2, n}^{i}$ are the coefficient functions which contribute to the structure functions $F_{L}^{\gamma}$ and $F_{2}^{\gamma}$, respectively, and $O_{i}^{\mu_{1} \cdots \mu_{n}}$ and $O_{i}^{\lambda \sigma \mu_{1} \cdots \mu_{n-2}}$ are spin- $n$ twist-2 operators (hereafter we often refer to $O_{i}^{\mu_{1} \cdots \mu_{n}}$ as $O_{i}^{n}$ ). The sum on $i$ runs over the possible twist- 2 operators and $\cdots$ represents other terms with irrelevant coefficient functions and operators. In fact, the relevant $O_{i}^{n}$ are flavor singlet quark $(\psi)$, gluon $(G)$, flavor nonsinglet quark $(N S)$ and photon $(\gamma)$ operators. It is noted that the operators $O_{i}^{n}$ are traceless and have totally symmetric Lorentz indices $\mu_{1} \cdots \mu_{n}\left(\lambda \sigma \mu_{1} \cdots \mu_{n-2}\right)$.

The spin-averaged matrix elements of these operators sandwiched by the photon states with momentum $p$ are expressed as

$$
\begin{aligned}
\left\langle\gamma(p)\left|O_{i}^{\mu_{1} \cdots \mu_{n}}\right| \gamma(p)\right\rangle_{\text {spin av }}= & A_{n}^{i}\left(\mu^{2}, P^{2}\right)\left\{p^{\mu_{1}} \cdots p^{\mu_{n}}\right. \\
& - \text { trace terms }\} \\
\equiv & A_{n}^{i}\left(\mu^{2}, P^{2}\right)\left\{p^{\mu_{1}} \cdots p^{\mu_{n}}\right\}_{n},
\end{aligned}
$$

where $i=\psi, G, N S, \gamma$, and $A_{n}^{i}\left(\mu^{2}, P^{2}\right)$ is the reduced photon matrix element with $\mu$ being the renormalization point which we choose at $\mu^{2}=P^{2}$. For $-p^{2}=P^{2} \gg \Lambda^{2}$, we can calculate $A_{n}^{i}\left(P^{2}\right)$ perturbatively. The $\left\{p^{\mu_{1}} \cdots p^{\mu_{n}}\right\}_{n}$ denotes the totally symmetric rank- $n$ tensor formed with the momentum $p$ alone and satisfies the traceless condition $g_{\mu_{i} \mu_{j}}\left\{p^{\mu_{1}} \cdots p^{\mu_{n}}\right\}_{n}=0$. Taking the spin-averaged photon matrix elements of (2.7) we obtain for the photon-photon forward-scattering amplitude

$$
\begin{aligned}
T_{\mu \nu}^{\gamma}(p, q)= & i \int d^{4} x e^{i q \cdot x}\left\langle\gamma(p)\left|T\left(J_{\mu}(x) J_{\nu}(0)\right)\right| \gamma(p)\right\rangle_{\text {spin av }} \\
= & \left(g_{\mu \nu}-\frac{q_{\mu} q_{\nu}}{q^{2}}\right) \sum_{\substack{n=0 \\
n=\text { even }}}\left(\frac{2}{Q^{2}}\right)^{n} q_{\mu_{1}} \cdots q_{\mu_{n}}\left\{p^{\mu_{1}} \cdots p^{\mu_{n}}\right\}_{n} \sum_{i} C_{L, n}^{i} A_{n}^{i}\left(P^{2}\right)+\left(-g_{\mu \lambda} g_{\nu \sigma} q^{2}+g_{\mu \lambda} q_{\nu} q_{\sigma}+g_{\nu \sigma} q_{\mu} q_{\lambda}\right. \\
& \left.-g_{\mu \nu} q_{\lambda} q_{\sigma}\right) \sum_{\substack{n=2 \\
n=\text { even }}}\left(\frac{2}{Q^{2}}\right)^{n} q_{\mu_{1}} \cdots q_{\mu_{n-2}}\left\{p^{\lambda} p^{\sigma} p^{\mu_{1}} \cdots p^{\mu_{n-2}}\right\}_{n} \sum_{i} C_{2, n}^{i} A_{n}^{i}\left(P^{2}\right) .
\end{aligned}
$$


The basic idea for treating target mass corrections exactly is to take account of trace terms in the traceless tensors properly. We evaluate the contraction between $q_{\mu_{1}} \cdots q_{\mu_{n}}$ and the traceless tensors without neglecting any of the trace terms. The results are expressed in terms of Gegenbauer polynomials $[25,28,29]$ :

$$
\begin{aligned}
& q_{\mu_{1}} \cdots q_{\mu_{n}}\left\{p^{\mu_{1}} \cdots p^{\mu_{n}}\right\}_{n}=a^{n} C_{n}^{(1)}(\eta) \\
q_{\mu_{1}} \cdots & q_{\mu_{n-2}}\left\{p^{\lambda} p^{\sigma} p^{\mu_{1}} \cdots p^{\left.\mu_{n-2}\right\}_{n}}\right. \\
= & \frac{1}{n(n-1)}\left[\frac{g^{\lambda \sigma}}{Q^{2}} a^{n} 2 C_{n-2}^{(2)}(\eta)+\frac{q^{\lambda} q^{\sigma}}{Q^{4}} a^{n} 8 C_{n-4}^{(3)}(\eta)\right. \\
& +p^{\lambda} p^{\sigma} a^{n-2} 2 C_{n-2}^{(3)}(\eta) \\
& \left.+\frac{p^{\lambda} q^{\sigma}+q^{\lambda} p^{\sigma}}{Q^{2}} a^{n-1} 4 C_{n-3}^{(3)}(\eta)\right]
\end{aligned}
$$

where

$$
a=-\frac{1}{2} P Q, \quad \eta=-\frac{p \cdot q}{P Q},
$$

and $C_{n}^{(\nu)}(\eta)$ 's are Gegenbauer polynomials (see Appendix B). Recall that in the case of a nucleon target with mass $M$, we had $p^{2}=M^{2}, \eta=i p \cdot q / M Q$ and $a=$ $-\frac{1}{2} i M Q$ [28]. In the photon case, we have $p^{2}=-P^{2}$ instead, and thus, replacing $M$ with $-i P$, we obtain the expressions for $a$ and $\eta$ in (2.12). The derivation of Eqs. (2.10) and (2.11) are given in Appendix C.

We decompose the amplitude $T_{\mu \nu}^{\gamma}(p, q)$ as

$$
\begin{aligned}
T_{\mu \nu}^{\gamma}(p, q)= & e_{\mu \nu}\left\{\frac{1}{x} T_{L}^{\gamma}+\frac{p^{2} q^{2}}{(p \cdot q)^{2}} \frac{1}{x}(p \cdot q) T_{2}^{\gamma}\right\} \\
& +d_{\mu \nu} \frac{1}{x}(p \cdot q) T_{2}^{\gamma},
\end{aligned}
$$

then, using the results (2.10) and (2.11), we find from Eq. (2.9)

$$
\frac{1}{x}(p \cdot q) T_{2}^{\gamma}=\sum_{\substack{n=2 \\ n=\text { even }}}\left(-\frac{P}{Q}\right)^{n} 16 \eta^{2} C_{n-2}^{(3)}(\eta) \frac{1}{n(n-1)} M_{2, n}^{\gamma},
$$

$$
\begin{aligned}
\frac{1}{x} T_{L}^{\gamma}+ & \frac{p^{2} q^{2}}{(p \cdot q)^{2}} \frac{1}{x}(p \cdot q) T_{2}^{\gamma} \\
= & \sum_{\substack{n=0 \\
n=\text { even }}}\left(-\frac{P}{Q}\right)^{n} 2 C_{n}^{(1)}(\eta) M_{L, n}^{\gamma}+\sum_{\substack{n=2 \\
n=\text { even }}}\left(-\frac{P}{Q}\right)^{n} \frac{8}{n(n-1)} \\
& \times\left[C_{n-2}^{(2)}(\eta)-2 C_{n-4}^{(3)}(\eta)+4 \eta C_{n-3}^{(3)}(\eta)\right] M_{2, n}^{\gamma},
\end{aligned}
$$

where we have defined

$$
\begin{aligned}
M_{2, n}^{\gamma} & \equiv \frac{1}{2} \sum_{i} C_{2, n}^{i}\left(Q^{2}, P^{2}, g\right) A_{n}^{i}\left(P^{2}\right), \\
M_{L, n}^{\gamma} & \equiv \frac{1}{2} \sum_{i} C_{L, n}^{i}\left(Q^{2}, P^{2}, g\right) A_{n}^{i}\left(P^{2}\right) .
\end{aligned}
$$

\section{NACHTMANN MOMENTS}

We derive the Nachtmann moments for the definite spin- $n$ contributions, $M_{2, n}^{\gamma}$ and $M_{L, n}^{\gamma}$. First we write the dispersion relations for $T_{2}^{\gamma}$ and $T_{L}^{\gamma}$, and we denote

$$
F_{2}^{\gamma}=\frac{1}{\pi} \operatorname{Im}(p \cdot q) T_{2}^{\gamma}, \quad F_{L}^{\gamma}=\frac{1}{\pi} \operatorname{Im} T_{L}^{\gamma} .
$$

Then, using the orthogonality relation (B4) and the integration formula (B5) for the Gegenbauer polynomials $C_{n}^{(\nu)}(\eta)$, we can project out $M_{2, n}^{\gamma}$ and $M_{L, n}^{\gamma}$. The results are as follows:

$$
\begin{aligned}
\mu_{2, n}^{\gamma}\left(Q^{2}, P^{2}\right) \equiv & \int_{0}^{x_{\max }} d x \frac{1}{x^{3}} \xi^{n+1} \\
& \times\left[\frac{3+3(n+1) r+n(n+2) r^{2}}{(n+2)(n+3)}\right] \\
& \times F_{2}^{\gamma}\left(x, Q^{2}, P^{2}\right) \\
= & M_{2, n}^{\gamma}, \\
\mu_{L, n}^{\gamma}\left(Q^{2}, P^{2}\right) \equiv & \int_{0}^{x_{\max }} d x \frac{1}{x^{3}} \xi^{n+1}\left[F_{L}^{\gamma}\left(x, Q^{2}, P^{2}\right)+\frac{4 P^{2} x^{2}}{Q^{2}}\right. \\
& \left.\times \frac{(n+3)-(n+1) \xi^{2} P^{2} / Q^{2}}{(n+2)(n+3)} F_{2}^{\gamma}\left(x, Q^{2}, P^{2}\right)\right] \\
= & M_{L, n}^{\gamma} .
\end{aligned}
$$

The Nachtmann moments $\mu_{2, n}^{\gamma}$ and $\mu_{L, n}^{\gamma}$ are given by the weighted integrals of the structure functions $F_{2}^{\gamma}$ and $F_{L}^{\gamma}$ and are equal to the definite spin- $n$ contributions, $M_{2, n}^{\gamma}$ and $M_{L, n}^{\gamma}$, respectively. The variables $r$ and $\xi$ are defined as

$$
r \equiv \sqrt{1-\frac{4 P^{2} x^{2}}{Q^{2}}}, \quad \xi \equiv \frac{2 x}{1+\sqrt{1-\frac{4 P^{2} x^{2}}{Q^{2}}}}=\frac{2 x}{1+r} .
$$

We see from Eq. (1.2) that the maximal value of $x$ is not 1 but $1 /\left[1+\left(P^{2} / Q^{2}\right)\right]$. Therefore, the allowed ranges of $r$ and $\xi$ turn out to be $r_{\min } \leq r \leq 1$ and $0 \leq \xi \leq 1$, respectively, where $r_{\min }=r\left(x_{\max }\right)=\left(1-P^{2} / Q^{2}\right) /\left(1+P^{2} / Q^{2}\right)$ and $\xi\left(x_{\max }\right)=1$.

We now outline how to derive the Nachtmann moments for the case of $F_{2}^{\gamma}\left(x, Q^{2}, P^{2}\right)$ given in (3.2). Since $(p \cdot q) / x=(-P Q \eta)(-P / Q) 2 \eta$, we see that $T_{2}^{\gamma}$ in (2.14) is expressed as 


$$
\begin{aligned}
T_{2}^{\gamma}\left(x, Q^{2}, P^{2}\right)= & \frac{1}{Q^{2}} \sum_{\substack{n=2 \\
n=\text { even }}}\left(-\frac{P}{Q}\right)^{n-2} \cdot 8 C_{n-2}^{(3)}(\eta) \\
& \times \frac{1}{n(n-1)} M_{2, n}^{\gamma} .
\end{aligned}
$$

By the use of orthogonality relation of the Gegenbauer polynomials (B4) for $\nu=3$ we get

$$
\begin{aligned}
& \int_{-1}^{1}\left(1-\eta^{2}\right)^{5 / 2} C_{n-2}^{(3)}(\eta) T_{2}^{\gamma} d \eta \\
& \quad=\frac{1}{Q^{2}} \frac{\pi}{2^{4}}\left(-\frac{P}{Q}\right)^{n-2}(n+2)(n+3) M_{2, n}^{\gamma} .
\end{aligned}
$$

Applying the dispersion relation, we can relate the full amplitude with its absorptive part:

$$
\begin{aligned}
T_{2}^{\gamma}(\omega) & =\int_{\omega_{\min }}^{\infty}\left(\frac{1}{\omega^{\prime}-\omega}+\frac{1}{\omega^{\prime}+\omega}\right) W_{2}^{\gamma}\left(\omega^{\prime}\right) d \omega^{\prime}, \\
\omega_{\min } & =\frac{1}{x_{\max }},
\end{aligned}
$$

where $\quad \omega=2 p \cdot q / Q^{2}=1 / x$ and denoting $\zeta=$ $\left(\omega^{\prime} Q\right) /(-2 P), \eta=(\omega Q) /(-2 P)$ we derive

$$
\begin{aligned}
\int_{-1}^{1}(1 & \left.-\eta^{2}\right)^{5 / 2} C_{n-2}^{(3)}(\eta) T_{2}^{\gamma} d \eta \\
= & 2 \int_{\omega_{\min }}^{\infty} d \omega^{\prime} W_{2}^{\gamma}\left(\omega^{\prime}, Q^{2}, P^{2}\right) \frac{Q}{(-2 P)} \\
& \times \int_{-1}^{1} \frac{(-1)}{(-\zeta)-\eta}\left(1-\eta^{2}\right)^{5 / 2} C_{n-2}^{(3)}(\eta) d \eta,
\end{aligned}
$$

where we have noted $C_{n-2}^{(3)}(-\eta)=C_{n-2}^{(3)}(\eta)$ for even $n$. From (B5) for $m=0, \nu=3$, we get

$$
\begin{aligned}
\int_{-1}^{1}(1 & \left.-\eta^{2}\right)^{5 / 2} C_{n-2}^{(3)}(\eta) T_{2}^{\gamma} d \eta \\
= & \left(\frac{-Q}{2 P}\right) \cdot \frac{\pi}{4} \cdot \int_{\omega_{\min }}^{\infty} d \omega W_{2}^{\gamma}\left(\omega, Q^{2}, P^{2}\right)\left(\zeta^{2}-1\right)(-1) \\
& \times\left[-\zeta-\left(\zeta^{2}-1\right)^{1 / 2}\right]^{n+1}(n+2)(n+3) \\
& \times\left[1-\frac{6}{n+2} z+\frac{12}{(n+2)(n+3)} z^{2}\right]
\end{aligned}
$$

where we used the following relation for the hypergeometric function with $\zeta=-p \cdot q / P Q$,

$$
\begin{aligned}
F(3,-2 ; n+2 ; z) & =1-\frac{6}{n+2} z+\frac{12}{(n+2)(n+3)} z^{2}, \\
z & \equiv \frac{-(-\zeta)+\left(\zeta^{2}-1\right)^{1 / 2}}{2\left(\zeta^{2}-1\right)^{1 / 2}} .
\end{aligned}
$$

Setting (3.6) equal to (3.9) and changing integration variable from $\omega$ to $x$, we get

$$
\begin{gathered}
\int_{0}^{x_{\max }} d x \frac{1}{x^{3}} \xi^{n+1}\left(1-\frac{4 P^{2} x^{2}}{Q^{2}}\right)\left[1-\frac{6}{n+2} z\right. \\
\left.+\frac{12}{(n+2)(n+3)} z^{2}\right] F_{2}^{\gamma}\left(x, Q^{2}, P^{2}\right)=M_{2, n}^{\gamma} .
\end{gathered}
$$

Here one should note that $n$ is even and the following relations hold:

$$
\begin{gathered}
-\zeta=\frac{Q}{2 P x}, \quad-\zeta-\left(\zeta^{2}-1\right)^{1 / 2}=\frac{P}{Q} \xi, \\
z=-\frac{P^{2}}{Q^{2}} \frac{\xi x}{r}, \quad \xi=\frac{Q^{2}}{2 P^{2} x}(1-r),
\end{gathered}
$$

and so we finally get for $F_{2}^{\gamma}\left(x, Q^{2}, P^{2}\right)$ as

$$
\begin{aligned}
\int_{0}^{x_{\max }} d x \frac{1}{x^{3}} \xi^{n+1} & {\left[\frac{3+3(n+1) r+n(n+2) r^{2}}{(n+2)(n+3)}\right] } \\
& \times F_{2}^{\gamma}\left(x, Q^{2}, P^{2}\right)=M_{2, n}^{\gamma}\left(Q^{2}, P^{2}\right),
\end{aligned}
$$

where the left-hand side is $\mu_{2, n}^{\gamma}\left(Q^{2}, P^{2}\right)$, the Nachtmann moment, which is equal to the definite spin- $n$ contribution $M_{2, n}^{\gamma}$, and this is consistent with the previous result for the case of nucleon target [25,34-36] with a replacement of the variable $M \rightarrow-i P$.

For the longitudinal structure function, we first solve for $\frac{1}{x} T_{L}^{\gamma}$ from Eqs. (2.14) and (2.15) and we get (see Appendix B)

$$
\begin{aligned}
\frac{1}{x} T_{L}^{\gamma}= & \sum_{\substack{n=0 \\
n=\text { even }}}\left(-\frac{P}{Q}\right)^{n} C_{n}^{(1)}(\eta) M_{L, n}^{\gamma}+\sum_{\substack{n=2 \\
n=\text { even }}}\left(-\frac{P}{Q}\right)^{n} \frac{4}{n(n-1)} \\
& \times\left[\frac{-2}{n} C_{n-2}^{(3)}(\eta)+\frac{2}{n} C_{n-4}^{(3)}(\eta)\right] M_{2, n}^{\gamma} .
\end{aligned}
$$

Then using the recursion relation (B10) for the case $\nu=1$ :

$$
\begin{aligned}
C_{n}^{(1)}(\eta)= & \frac{2}{(n+1)(n+2)} C_{n}^{(3)}(\eta)-\frac{4}{n(n+2)} C_{n-2}^{(3)}(\eta) \\
& +\frac{2}{n(n+1)} C_{n-4}^{(3)}(\eta),
\end{aligned}
$$

and the orthogonality relation of the Gegenbauer polynomials $C_{n}^{(3)}$ 's we can derive the recursive relations for the sequence $M_{L, n}^{\gamma}$ 's which can be solved as (See Appendix D):

$$
\begin{aligned}
& \int_{0}^{x_{\max }} d x \frac{1}{x^{3}} \xi^{n+1}\left[F_{L}^{\gamma}\left(x, Q^{2}, P^{2}\right)+\frac{4 P^{2} x^{2}}{Q^{2}}\right. \\
& \left.\quad \times \frac{(n+3)-(n+1) \xi^{2} P^{2} / Q^{2}}{(n+2)(n+3)} F_{2}^{\gamma}\left(x, Q^{2}, P^{2}\right)\right] \\
& =M_{L, n}^{\gamma},
\end{aligned}
$$

where the left-hand side integral is the Nachtmann moment $\mu_{L, n}^{\gamma}$ for the longitudinal part. This coincides with the result obtained in $[25,34]$ after the replacement mentioned above. 


\section{INVERTING THE NACHTMANN MOMENTS}

We now invert the Nachtmann moments to express the structure functions $F_{2}^{\gamma}$ and $F_{L}^{\gamma}$ explicitly as functions of $x$, $Q^{2}$ and $P^{2}$. We first consider $F_{2}^{\gamma}$. By changing the integration variable from $x$ to $\xi$, we can rewrite the Nachtmann moments, given in Eqs. (3.2) and (3.3), as follows:

$$
\begin{aligned}
M_{2, n}^{\gamma}= & \int_{0}^{1} d \xi \xi^{n-2}\left(1-\kappa \xi^{2}\right)\left(1+\kappa \xi^{2}\right)\left[\frac{3}{(n+2)(n+3)}\right. \\
& \left.+\frac{3(n+1)}{(n+2)(n+3)} \frac{1-\kappa \xi^{2}}{1+\kappa \xi^{2}}+\frac{n}{n+3}\left(\frac{1-\kappa \xi^{2}}{1+\kappa \xi^{2}}\right)^{2}\right] \\
& \times F_{2}^{\gamma}\left(x, Q^{2}, P^{2}\right), \\
M_{L, n}^{\gamma}= & \int_{0}^{1} d \xi \xi^{n-2}\left(1-\kappa \xi^{2}\right)\left(1+\kappa \xi^{2}\right)\left[F_{L}^{\gamma}\left(x, Q^{2}, P^{2}\right)\right. \\
& +4 \kappa \frac{\xi^{2}}{\left(1+\kappa \xi^{2}\right)^{2}}\left(\frac{1}{n+2}-\kappa \xi^{2} \frac{n+1}{(n+2)(n+3)}\right) \\
& \left.\times F_{2}^{\gamma}\left(x, Q^{2}, P^{2}\right)\right],
\end{aligned}
$$

where we have made use of the following relations:

$$
x=\frac{\xi}{1+\kappa \xi^{2}}, \quad r=\frac{1-\kappa \xi^{2}}{1+\kappa \xi^{2}}, \quad \frac{d x}{d \xi}=\frac{1-\kappa \xi^{2}}{\left(1+\kappa \xi^{2}\right)^{2}},
$$

with $\kappa=P^{2} / Q^{2}$. Now we define

$$
\begin{aligned}
& A(\xi) \equiv \frac{1-\kappa \xi^{2}}{1+\kappa \xi^{2}} F_{2}^{\gamma}\left(x, Q^{2}, P^{2}\right), \\
& B(\xi) \equiv\left(1-\kappa \xi^{2}\right)\left(1+\kappa \xi^{2}\right) F_{L}^{\gamma}\left(x, Q^{2}, P^{2}\right) .
\end{aligned}
$$

Then the above two moments are written as

$$
\begin{aligned}
\frac{M_{2, n}^{\gamma}}{n(n-1)}= & \int_{0}^{1} d \xi \xi^{n-2}\left\{\frac{A(\xi)}{n(n-1)}-\frac{2 \kappa \xi^{2} A(\xi)}{n(n+2)}\right. \\
& \left.+\frac{\kappa^{2} \xi^{4} A(\xi)}{(n+2)(n+3)}\right\}, \\
\frac{M_{L, n}^{\gamma}}{n+1}= & \int_{0}^{1} d \xi \xi^{n-2}\left\{\frac{B(\xi)}{n+1}+\frac{4 \kappa \xi^{2} A(\xi)}{(n+1)(n+2)}\right. \\
& \left.-\frac{4 \kappa^{2} \xi^{4} A(\xi)}{(n+2)(n+3)}\right\} .
\end{aligned}
$$

The boundary conditions for $A(\xi)$ and $B(\xi)$ are $A(\xi=1)=B(\xi=1)=0, \quad$ since $\quad F_{2}^{\gamma}\left(x_{\max }, Q^{2}, P^{2}\right)=$ $F_{L}^{\gamma}\left(x_{\max }, Q^{2}, P^{2}\right)=0$ and $\xi\left(x_{\max }\right)=1$. Now introducing the following four functions,

$$
\begin{gathered}
A_{0}(\xi)=\int_{\xi}^{1} d \xi^{\prime} A\left(\xi^{\prime}\right), \quad A_{-1}(\xi) \equiv \int_{\xi}^{1} d \xi^{\prime} \frac{A\left(\xi^{\prime}\right)}{\xi^{\prime}}, \\
A_{-2}(\xi)=\int_{\xi}^{1} d \xi^{\prime} \frac{A\left(\xi^{\prime}\right)}{\xi^{\prime 2}}
\end{gathered}
$$

$$
B_{-3}(\xi) \equiv \int_{\xi}^{1} d \xi^{\prime} \frac{B\left(\xi^{\prime}\right)}{\xi^{\prime 3}},
$$

and by partial integration we find that the above two moments are written as

$$
\begin{aligned}
\frac{M_{2, n}^{\gamma}}{n(n-1)}= & \int_{0}^{1} d \xi \xi^{n-2}\left(1-\kappa \xi^{2}\right)\left\{\left(1+\kappa \xi^{2}\right) A_{-1}(\xi)\right. \\
& \left.-\xi A_{-2}(\xi)-\kappa \xi A_{0}(\xi)\right\}, \\
\frac{M_{L, n}^{\gamma}}{n+1}= & \int_{0}^{1} d \xi \xi^{n}\left[B_{-3}(\xi)+4 \kappa\left\{\left(1+\kappa \xi^{2}\right) A_{-1}(\xi)\right.\right. \\
& \left.\left.-\xi A_{-2}(\xi)-\kappa \xi A_{0}(\xi)\right\}\right] .
\end{aligned}
$$

Inverting the moments we get

$$
\begin{aligned}
G(\xi)= & \frac{1}{2 \pi i} \int_{c-i \infty}^{c+i \infty} d n \xi^{-n+1}\left\{\frac{M_{2, n}^{\gamma}}{n(n-1)}\right\} \\
= & \left(1-\kappa \xi^{2}\right)\left\{\left(1+\kappa \xi^{2}\right) A_{-1}(\xi)-\xi A_{-2}(\xi)\right. \\
& \left.-\kappa \xi A_{0}(\xi)\right\},
\end{aligned}
$$

$$
\begin{aligned}
S(\xi) & =\frac{1}{2 \pi i} \int_{c-i \infty}^{c+i \infty} d n \xi^{-n-1}\left\{\frac{M_{L, n}^{\gamma}}{n+1}\right\} \\
& =B_{-3}(\xi)+\frac{4 \kappa}{1-\kappa \xi^{2}} G(\xi),
\end{aligned}
$$

where in Eq. (4.12) we have used the result of Eq. (4.11).

We further introduce the following functions,

$$
\begin{gathered}
H(\xi) \equiv-\frac{d G(\xi)}{d \xi}=\frac{1}{2 \pi i} \int_{c-i \infty}^{c+i \infty} d n \xi^{-n} \frac{M_{2, n}^{\gamma}}{n}, \\
F(\xi) \equiv-\frac{d H(\xi)}{d \xi}=\frac{1}{2 \pi i} \int_{c-i \infty}^{c+i \infty} d n \xi^{-n-1} M_{2, n}^{\gamma}, \\
F_{L}(\xi) \equiv-\xi \frac{d S(\xi)}{d \xi}=\frac{1}{2 \pi i} \int_{c-i \infty}^{c+i \infty} d n \xi^{-n-1} M_{L, n}^{\gamma} .
\end{gathered}
$$

Differentiating both sides of Eqs. (4.11) and (4.12) with respect to $\xi$, we get the relations between $A_{-1}(\xi)$, $\left(A_{-2}(\xi)+\kappa A_{0}(\xi)\right), A(\xi), B(\xi)$ and $G(\xi), H(\xi), F_{2}(\xi)$ and $F_{L}(\xi)$. Now solving for $A(\xi)$ and $B(\xi)$ and, then recalling Eq. (4.4), we obtain

$$
\begin{aligned}
& F_{2}^{\gamma}\left(x, Q^{2}, P^{2}\right)=\frac{x^{2}}{r^{3}} F(\xi)-6 \kappa \frac{x^{3}}{r^{4}} H(\xi)+12 \kappa^{2} \frac{x^{4}}{r^{5}} G(\xi), \\
& F_{L}^{\gamma}\left(x, Q^{2}, P^{2}\right)=\frac{x^{2}}{r} F_{L}(\xi)-4 \kappa \frac{x^{3}}{r^{2}} H(\xi)+8 \kappa^{2} \frac{x^{4}}{r^{3}} G(\xi) .
\end{aligned}
$$

Equations (4.16) and (4.17) are the final formulas for the photon structure functions $F_{2}^{\gamma}$ and $F_{L}^{\gamma}$ when target mass effects are taken into account. They can also be derived 
from the method of Georgi and Politzer [26]. Once $M_{2, n}^{\gamma}$ and $M_{L, n}^{\gamma}$ in Eq. (2.16), are given, then we can calculate the four "profile" functions $G(\xi), H(\xi), F(\xi)$ and $F_{L}(\xi)$ through Eqs. (4.11), (4.13), (4.14), and (4.15), and by using Eqs. (4.16) and (4.17) we can predict whole structure functions with target mass corrections.

Note that in the above expressions, the $Q^{2}$ - as well as $P^{2}$ dependence of $F(\xi), G(\xi), H(\xi)$, and $F_{L}(\xi)$ are implicit, since they are given by $M_{2, n}^{\gamma}$ and $M_{L, n}^{\gamma}$ which depend on $Q^{2}$ as well as on $P^{2}$. If we take the $\kappa \rightarrow 0$ limit, the above expression reduces to that for the $F_{2}^{\gamma}$ and $F_{L}^{\gamma}$ without TME. And in the absence of TME we have

$$
\begin{aligned}
& M_{2, n}^{\gamma}=\int_{0}^{1} d x x^{n-2} F_{2}^{\gamma}\left(x, Q^{2}, P^{2}\right), \\
& M_{L, n}^{\gamma}=\int_{0}^{1} d x x^{n-2} F_{L}^{\gamma}\left(x, Q^{2}, P^{2}\right) .
\end{aligned}
$$

From our previous QCD calculation [21] of $M_{2, n}^{\gamma}$ and $M_{L, n}^{\gamma}$ we already know the three functions, $F(\xi), G(\xi)$, and $H(\xi)$ to NNLO and $F_{L}(\xi)$ to NLO, so we can evaluate the photon structure functions with TME to the same accuracies as in the case we neglect TME.

\section{NUMERICAL ANALYSIS}

In this section we perform a numerical analysis for the structure functions $F_{2}^{\gamma}$ and $F_{L}^{\gamma}$ when TME are included. We first compute the four profile functions $G, H, \mathrm{~F}$, and $F_{L}$ which are given in Eq. (4.11), (4.13), (4.14), and (4.15). We use the QCD results for $M_{2, n}^{\gamma}$ and $M_{L, n}^{\gamma}$, which have been calculated up to the NNLO and the NLO in QCD, respectively, in Ref. [21]. Indeed, the expressions of $M_{2, n}^{\gamma}$ and $M_{L, n}^{\gamma}$ are given in the right-hand sides of Eq. (2.29) and Eq. (6.3) of Ref. [21]. In Fig. 3 we have plotted the functions $F, G, H$ and $F_{L}$ as functions of $x$ for the case of $Q^{2}=30 \mathrm{GeV}^{2}$ and $P^{2}=1 \mathrm{GeV}^{2}$ with $x_{\max }=0.968$.

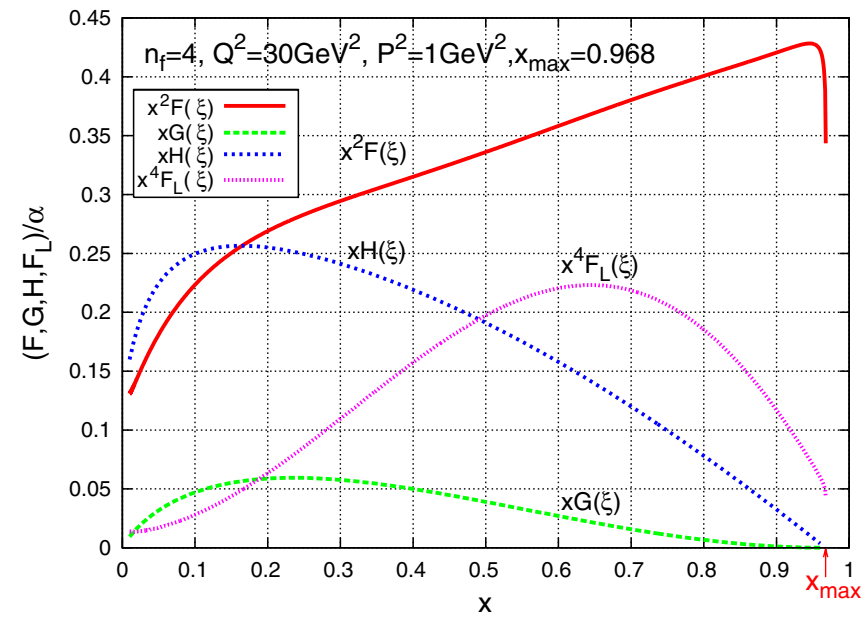

FIG. 3 (color online). The four functions $F, G, H$ and $F_{L}$ as functions of $x . Q^{2}=30 \mathrm{GeV}^{2}$ and $P^{2}=1 \mathrm{GeV}^{2} \cdot x_{\max }=0.968$.

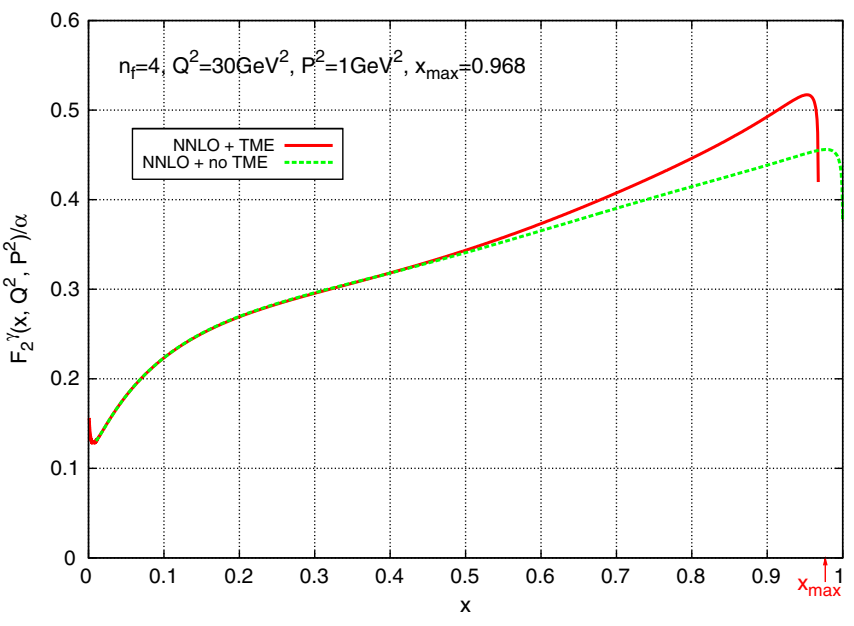

FIG. 4 (color online). $\quad F_{2}^{\gamma}\left(x, Q^{2}, P^{2}\right)$ as a function of $x$ for $Q^{2}=$ $30 \mathrm{GeV}^{2}$ and $P^{2}=1 \mathrm{GeV}^{2}$ with $x_{\max }=0.968$.

We take $\Lambda=0.2 \mathrm{GeV}$ for the QCD parameter and $n_{f}=4$ for the number of active quark flavors throughout our numerical analysis. Note that we have multiplied each function by a suitable power of $x$ to accommodate four functions in a single graph. The Bjorken variable $x$ ranges from 0 to $x_{\max }$.

Now inserting the functions $F, G, H$ and $F_{L}$ into Eqs. (4.16) and (4.17), we obtain the graphs of $F_{2}^{\gamma}\left(x, Q^{2}, P^{2}\right)$ and $F_{L}^{\gamma}\left(x, Q^{2}, P^{2}\right)$ as functions of $x$, which are shown in Figs. 4 and 5, respectively. We observe that TME become sizable at larger $x$ region. While TME enhances $F_{2}^{\gamma}$ at larger $x$, it reduces $F_{L}^{\gamma}$. In fact, $F_{2}^{\gamma}$ becomes maximum at $x$ very close to the maximal value of $x, x_{\max }$ (1) for the case with (without) TME. The target mass correction is of order $10 \%$ when compared at the maximal values for $F_{2}^{\gamma}$. In the case of $F_{L}^{\gamma}$, the maximal value is attained in the middle $x$, where the TME reduces the $F_{L}^{\gamma}$ about $5 \%$.

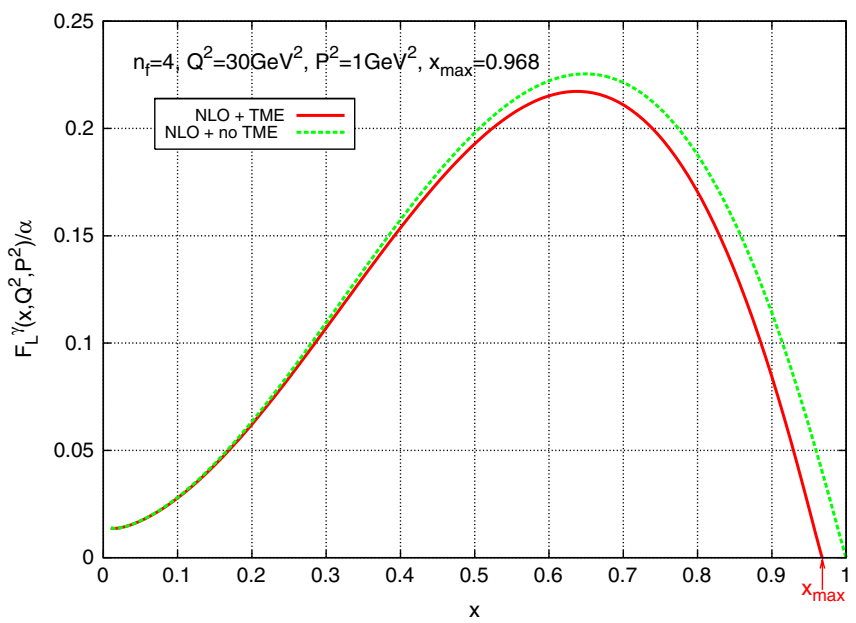

FIG. 5 (color online). $\quad F_{L}^{\gamma}\left(x, Q^{2}, P^{2}\right)$ as a function of $x$ for $Q^{2}=$ $30 \mathrm{GeV}^{2}$ and $P^{2}=1 \mathrm{GeV}^{2}$ with $x_{\max }=0.968$. 
One should note that $F(\xi)$ is dominant at larger $x$ region, and determines the leading behavior of $F_{2}^{\gamma}$. And the factor $x^{2} / r^{3}$ in front of $F_{2}^{\gamma}$ shows the deviation upwards from $x^{2}$ as $x$ approaches $x_{\max }$. On the other hand, the expression for $F_{L}^{\gamma}$, Eq. (4.17), possesses no dependence upon the function $F(\xi)$, in contrast to the case of $F_{2}^{\gamma}$. This is a reason why $F_{L}^{\gamma}$ becomes maximum in the middle $x$ region, as seen from Fig. 3.

Now let us compare our theoretical prediction for the virtual photon structure functions with the existing experimental data. In Fig. 6 and 7, we have plotted the experimental data from PLUTO Collaboration [37] and also those from L3 Collaboration [38] on the so-called "effective photon structure function" defined as $F_{\text {eff }}^{\gamma}=$ $F_{2}^{\gamma}+\frac{3}{2} F_{L}^{\gamma}$, together with the theoretical predictions. The effective structure function is proportional to $\sigma_{T T}+$ $\sigma_{L T}+\sigma_{T L}+\sigma_{L L}$, where $\sigma_{a b} \quad(a, b=T, \quad L ; \quad T=$ transverse and $L=$ longitudinal) is the total cross section with the helicity state (a) of the probe photon and helicity (b) of the target photon. This combination of $F_{2}^{\gamma}$ and $F_{L}^{\gamma}$ is obtained in the limit of $P^{2} / Q^{2} \ll 1[10,39,40]$.

In the above experiments we have $Q^{2}=5(120) \mathrm{GeV}^{2}$ and $P^{2}=0.35(3.7) \mathrm{GeV}^{2}$ with $x_{\max }=0.93(0.97)$, for PLUTO (L3) data. Here we note that for the PLUTO data, $P^{2} \ll Q^{2}$ is satisfied since $P^{2} / Q^{2} \simeq 0.07$, but $P^{2}$ is not much larger than $\Lambda^{2}$ i.e. $\Lambda^{2} / P^{2} \simeq 0.114$. For L3 data, both hierarchical conditions are satisfied. Although the experimental error bars are rather large, the data are considered to be roughly consistent with the theoretical expectations, except for the larger $x$ region in the case of L3 data. Note that the TME for the $F_{\text {eff }}^{\gamma}$ in this kinematical region is almost negligible for both cases. This could be

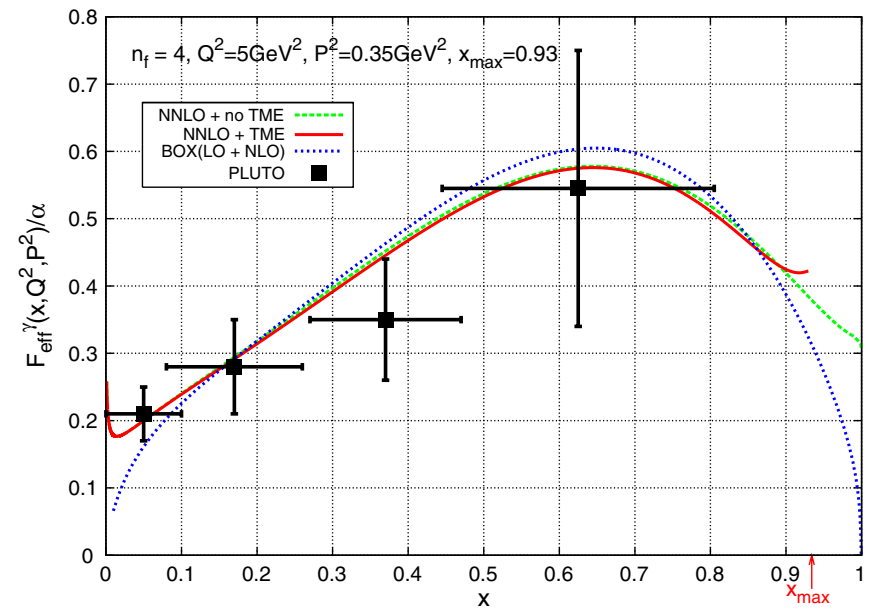

FIG. 6 (color online). NNLO predictions with and without TME for the effective photon structure function: $F_{\text {eff }}^{\gamma}=F_{2}^{\gamma}+$ $\frac{3}{2} F_{L}^{\gamma}$ for $Q^{2}=5 \mathrm{GeV}^{2}$ and $P^{2}=0.35 \mathrm{GeV}^{2}$ with $x_{\max }=0.93$. The experimental data are from the PLUTO group [37]. The box diagram prediction to the NLO order is also shown (blue shortdotted line).

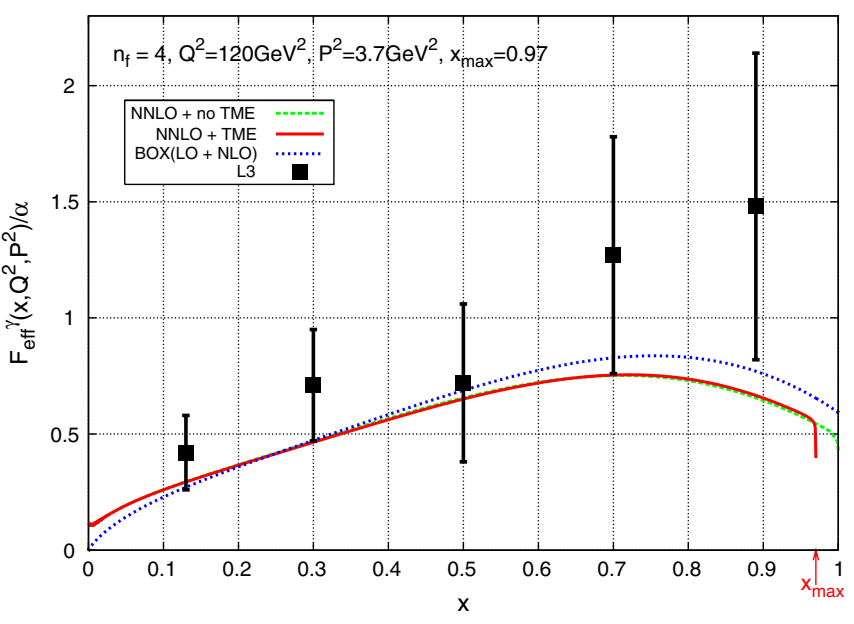

FIG. 7 (color online). NNLO predictions with and without TME and box prediction (NLO) on $F_{\text {eff }}^{\gamma}=F_{2}^{\gamma}+\frac{3}{2} F_{L}^{\gamma}$ for $Q^{2}=$ $120 \mathrm{GeV}^{2}$ and $P^{2}=3.7 \mathrm{GeV}^{2}$ with $x_{\max }=0.970$. The data are from the L3 Group [38].

explained as a consequence of the cancellation of the TME between $F_{2}^{\gamma}$ and $F_{L}^{\gamma}$, as discussed above.

\section{CONCLUSIONS}

We have investigated the target mass corrections for the unpolarized virtual photon structure functions $F_{2}^{\gamma}\left(x, Q^{2}, P^{2}\right)$ and $F_{L}^{\gamma}\left(x, Q^{2}, P^{2}\right)$ to the NNLO in perturbative QCD.

In contrast to the case of the nucleon target, the virtual photon target provides us with the unique testing ground for the perturbatively calculable target mass effects. Taking into account the trace terms in the operator matrix elements by using the expansion in terms of orthogonal Gegenbauer polynomials we get the Nachtmann moments. These moments were then inverted to derive explicit expressions for $F_{2}^{\gamma}\left(x, Q^{2}, P^{2}\right)$ and $F_{L}^{\gamma}\left(x, Q^{2}, P^{2}\right)$ in terms of the four profile functions which are calculable to NNLO for the first three functions $F, G, H$ and to NLO for the last one $F_{L}$. The TME becomes sizable at larger $x$ region, and it enlarges $F_{2}^{\gamma}$ near $x_{\max }$ and reduces $F_{L}^{\gamma}$ in the region of $x$ larger than the middle point. When we go to higher values of $P^{2}$, e.g. $P^{2}=$ $3 \mathrm{GeV}^{2}$ for $Q^{2}=30 \mathrm{GeV}^{2}$, it has turned out that the $F_{2}^{\gamma}$ blows up as $x$ approaches $x_{\max }$. So some prescription like resummation of large logs would be needed to avoid such difficulties.

We have carried out the confrontation of our theoretical predictions with the existing experimental data on the effective photon structure function $F_{\text {eff }}^{\gamma}$ from PLUTO and also those from L3 Collaboration. Roughly speaking we find the rather good agreement between theory and experiments. However, it turned out that TME looks almost negligible for $F_{\text {eff }}^{\gamma}$, which is the combination of $F_{2}^{\gamma}$ and $F_{L}^{\gamma}$ and exhibits a cancellation of TME between them. In the present analysis, we have treated the active flavors as 
massless quarks, and ignored the mass effects of the heavy flavors, which should remain as a future subject. We should also investigate the power corrections $\left(P^{2} / Q^{2}\right)^{k}(k=$ $1,2, \cdots)$ due to the higher-twist effects.

We expect the future experiments would provide us with more accurate data for the double-tag two-photon processes in $e^{+} e^{-}$collisions.

\section{ACKNOWLEDGMENTS}

One of the authors (T. Uematsu) would like to thank Guido Altarelli and Silvano Simula for the useful discussions. This research is supported in part by Grant-in-Aid for Scientific Research from the Ministry of Education, Culture, Sports, Science and Technology, Japan No. 18540267.

\section{APPENDIX A: PHOTON STRUCTURE FUNCTIONS}

Averaging the structure tensor $W_{\mu \nu \rho \tau}(p, q)$ given in Eq. (2.2) over the target polarization, we get $[41,42]$

$$
\begin{aligned}
W_{\mu \nu}^{\gamma}(p, q)= & -\frac{g^{\rho \tau}}{2} W_{\mu \nu \rho \tau}(p, q) \\
= & R_{\mu \nu}\left[W_{T T}-\frac{1}{2} W_{T L}\right] \\
& +k_{1 \mu} k_{1 \nu}\left[W_{L T}-\frac{1}{2} W_{L L}\right]
\end{aligned}
$$

where

$$
\begin{gathered}
R_{\mu \nu}=-g_{\mu \nu}+\frac{1}{X}\left[p \cdot q\left(q_{\mu} p_{\nu}+p_{\mu} q_{\nu}\right)\right. \\
\left.-q^{2} p_{\mu} p_{\nu}-p^{2} q_{\mu} q_{\nu}\right], \\
k_{1 \mu}=\sqrt{\frac{-q^{2}}{X}}\left(p_{\mu}-\frac{p \cdot q}{q^{2}} q_{\mu}\right),
\end{gathered}
$$

with $X=(p \cdot q)^{2}-q^{2} p^{2}$. In the above equation the first index $(a=T, L)$ of the invariant functions $W_{a b}$ refers to the probe photon and the second one $(b=T, L)$ to the target photon, and the subscripts $T$ and $L$ denote the transverse and longitudinal photon, respectively.

We define the unpolarized photon structure functions $F_{2}^{\gamma}$ and $F_{L}^{\gamma}$ as,

$$
\begin{gathered}
\frac{1}{x} F_{2}^{\gamma}=\frac{(p \cdot q)^{2}}{X}\left\{\left[W_{T T}-\frac{1}{2} W_{T L}\right]+\left[W_{L T}-\frac{1}{2} W_{L L}\right]\right\}, \\
\frac{1}{x} F_{L}^{\gamma}=W_{L T}-\frac{1}{2} W_{L L} .
\end{gathered}
$$

Another structure function $F_{1}^{\gamma}$ is often used, which is defined as [43]

$$
F_{1}^{\gamma}=W_{T T}-\frac{1}{2} W_{T L}
$$

Then we get a well-known relation

$$
F_{L}^{\gamma}=-x F_{1}^{\gamma}+\frac{X}{(p \cdot q)^{2}} F_{2}^{\gamma}=-x F_{1}^{\gamma}+\left(1-\frac{4 x^{2} P^{2}}{Q^{2}}\right) F_{2}^{\gamma} .
$$

Since $R_{\mu \nu}$ and $k_{1 \mu} k_{1 \nu}$ are expressed in terms of $e_{\mu \nu}$ and $d_{\mu \nu}$, which are given in Eqs. (2.5) and (2.6), as

$$
\begin{aligned}
& R_{\mu \nu}=\frac{p^{2} q^{2}}{X} e_{\mu \nu}+\frac{(p \cdot q)^{2}}{X} d_{\mu \nu}, \\
& k_{1 \mu} k_{1 \nu}=\frac{(p \cdot q)^{2}}{X}\left\{e_{\mu \nu}+d_{\mu \nu}\right\},
\end{aligned}
$$

we find that $W_{\mu \nu}^{\gamma}(p, q)$ in (A1) is rewritten as

$$
W_{\mu \nu}^{\gamma}(p, q)=e_{\mu \nu}\left\{\frac{1}{x} F_{L}^{\gamma}+\frac{p^{2} q^{2}}{(p \cdot q)^{2}} \frac{1}{x} F_{2}^{\gamma}\right\}+d_{\mu \nu} \frac{1}{x} F_{2}^{\gamma} .
$$

\section{APPENDIX B: GEGENBAUER POLYNOMIALS}

The Gegenbauer polynomials are defined through the generating function given by $[44,45]$

$$
\left(1-2 \eta t+t^{2}\right)^{-\nu}=\sum_{n=0}^{\infty} C_{n}^{(\nu)}(\eta) t^{n}
$$

In terms of hypergeometric functions $F(\alpha, \beta ; \gamma ; z), C_{n}^{(\nu)}(\eta)$ is expressed as

$$
\begin{aligned}
C_{n}^{(\nu)}(\eta) & =\frac{2^{n} \Gamma(n+\nu)}{n ! \Gamma(\nu)} \eta^{n} F\left(-\frac{n}{2}, \frac{1-n}{2} ; 1-n-\nu ; \frac{1}{\eta^{2}}\right) \\
& =\frac{1}{\Gamma(\nu)} \sum_{j=0}^{n / 2} \frac{(-1)^{j} \Gamma(\nu+n-j)}{j !(n-2 j) !}(2 \eta)^{n-2 j} .
\end{aligned}
$$

For example, we have

$$
C_{n}^{(1)}(\eta)=\sum_{j=0}^{n / 2} \frac{(-1)^{j}}{j !} \frac{(n-j) !}{(n-2 j) !}(2 \eta)^{n-2 j}
$$

\section{Orthogonality relations}

The orthogonality relation reads

$$
\begin{gathered}
\int_{-1}^{1}\left(1-\eta^{2}\right)^{\nu-(1 / 2)} C_{m}^{(\nu)}(\eta) C_{n}^{(\nu)}(\eta) d \eta \\
=\frac{2 \pi}{2^{2 \nu}} \frac{\Gamma(n+2 \nu)}{(n+\nu) n ![\Gamma(\nu)]^{2}} \delta_{m n} .
\end{gathered}
$$

In addition we have the following formula for the integral to project out the contributions of definite spin from the dispersion relations, 


$$
\begin{aligned}
\int_{-1}^{1} d \eta \eta^{m}\left(1-\eta^{2}\right)^{\nu-(1 / 2)} C_{n}^{(\nu)}(\eta) \frac{1}{\zeta-\eta} \\
=\frac{\pi}{2^{\nu-1}} \zeta^{m}\left(\zeta^{2}-1\right)^{(\nu-1) / 2}\left[\zeta-\left(\zeta^{2}-1\right)^{1 / 2}\right]^{n+\nu} \\
\quad \times \frac{\Gamma(n+2 \nu)}{\Gamma(\nu) \Gamma(n+\nu+1)} \\
\quad \times F\left(\nu, 1-\nu ; n+\nu+1 ; \frac{-\zeta+\left(\zeta^{2}-1\right)^{1 / 2}}{2\left(\zeta^{2}-1\right)^{1 / 2}}\right) .
\end{aligned}
$$

In fact the factor $\left[\zeta-\left(\zeta^{2}-1\right)^{1 / 2}\right]^{n+\nu}$ gives $\left(-\frac{P}{Q}\right)^{n+\nu} \xi^{n+\nu}$, where $\xi$ is the so-called $\xi$-scaling variable given in Eq. (3.4).

\section{Recursion relations} read

The recursion relations for Gegenbauer polynomials

$$
\begin{gathered}
n C_{n}^{(\nu)}(\eta)=2 \nu\left[\eta C_{n-1}^{(\nu+1)}(\eta)-C_{n-2}^{(\nu+1)}(\eta)\right], \\
(n+2 \nu) C_{n}^{(\nu)}(\eta)=2 \nu\left[C_{n}^{(\nu+1)}(\eta)-\eta C_{n-1}^{(\nu+1)}(\eta)\right], \\
(n+2) C_{n+2}^{(\nu)}(\eta)=2(n+\nu+1) \eta C_{n+1}^{(\nu)}(\eta) \\
-(n+2 \nu) C_{n}^{(\nu)}(\eta) .
\end{gathered}
$$

We get from (B6) and (B7),

$$
\begin{aligned}
C_{n}^{(\nu)}(\eta)= & \frac{\nu}{n+\nu}\left[C_{n}^{(\nu+1)}(\eta)-C_{n-2}^{(\nu+1)}(\eta)\right], \\
C_{n}^{(\nu)}(\eta)= & \frac{\nu}{n+\nu} \cdot \frac{\nu+1}{n+\nu+1} C_{n}^{(\nu+2)}(\eta) \\
& -\frac{2 \nu(\nu+1)}{(n+\nu)^{2}-1} C_{n-2}^{(\nu+2)}(\eta) \\
& +\frac{\nu}{n+\nu} \cdot \frac{\nu+1}{n+\nu-1} C_{n-4}^{(\nu+2)}(\eta) .
\end{aligned}
$$

Now we derive the second line of Eq. (3.14). Choosing $\nu=2$ and $n \rightarrow(n-2)$ in (B9), we find

$$
C_{n-2}^{(2)}(\eta)=\frac{2}{n}\left[C_{n-2}^{(3)}(\eta)-C_{n-4}^{(3)}(\eta)\right] .
$$

Next choosing $\nu=3$ and $n \rightarrow(n-4)$ in (B8), we get

$$
4 \eta C_{n-3}^{(3)}(\eta)=\frac{2}{n}\left[(n-2) C_{n-2}^{(3)}(\eta)+(n+2) C_{n-4}^{(3)}(\eta)\right] .
$$

Thus we obtain

$$
\begin{gathered}
{\left[C_{n-2}^{(2)}(\eta)-2 C_{n-4}^{(3)}(\eta)+4 \eta C_{n-3}^{(3)}(\eta)\right]-2 C_{n-2}^{(3)}(\eta)} \\
=\frac{-2}{n} C_{n-2}^{(3)}(\eta)+\frac{2}{n} C_{n-4}^{(3)}(\eta) .
\end{gathered}
$$

\section{APPENDIX C: CONTRACTION FORMULAS}

Here we derive Eqs. (2.10) and (2.11). The most general rank- $n$ symmetric and traceless tensor, $\left\{p^{\mu_{1}} \cdots p^{\mu_{n}}\right\}_{n}$, that can be formed with the momentum $p$ alone, is expressed as follows [26],

$$
\left\{p^{\mu_{1}} \cdots p^{\mu_{n}}\right\}_{n}=\sum_{j=0}^{n / 2} \frac{(-1)^{j}}{2^{j}} \frac{(n-j) !}{n !} \underbrace{g \cdots g}_{j} \overbrace{p \cdots p}^{n-2 j}\left(p^{2}\right)^{j},
$$

where $\underbrace{g \cdots g}_{j}$ stands for a product of $j$ metric tensors $g^{\mu_{l} \mu_{k}}$ with $2 j$ indices chosen among $\mu_{1}, \cdots, \mu_{n}$ in all possible ways. Then we easily see that the contraction of $\left\{p^{\mu_{1}} \cdots p^{\mu_{n}}\right\}_{n}$ with $q_{\mu_{1}} \cdots q_{\mu_{n}}$ is expressed in terms of Gegenbauer polynomial $C_{n}^{(1)}(\eta)$ given in (B3) as [25,28],

$$
q_{\mu_{1}} \cdots q_{\mu_{n}}\left\{p^{\mu_{1}} \cdots p^{\mu_{n}}\right\}=a^{n} C_{n}^{(1)}(\eta),
$$

which is Eq. (2.10). Here we have put

$$
a=-\frac{1}{2} P Q, \quad \eta=-\frac{p \cdot q}{P Q} .
$$

Next we differentiate both sides of Eq. (C2) twice with respect to $q_{\alpha}$ and $q_{\beta}$. The left-hand side becomes

$$
\begin{aligned}
& \frac{\partial}{\partial q_{\alpha}} \frac{\partial}{\partial q_{\beta}}\left(q_{\mu_{1}} \cdots q_{\mu_{n}}\left\{p^{\mu_{1}} \cdots p^{\left.\mu_{n}\right\}_{n}}\right)\right. \\
& \quad=n(n-1) q_{\mu_{1}} \cdots q_{\mu_{n-2}}\left\{p^{\alpha} p^{\beta} p^{\mu_{1}} \cdots p^{\left.\mu_{n-2}\right\}_{n} .}\right.
\end{aligned}
$$

Differentiation of $a^{n} C_{n}^{(1)}(\eta)$ with respect to $q_{\alpha}$ gives

$$
\begin{aligned}
\frac{\partial}{\partial q_{\alpha}}\left(a^{n} C_{n}^{(1)}(\eta)\right)= & n a^{n-1}\left(\frac{-a q^{\alpha}}{Q^{2}}\right) C_{n}^{(1)}(\eta) \\
& +a^{n} 2 C_{n-1}^{(2)}(\eta)\left(\frac{p^{\alpha}}{2 a}+\eta \frac{q^{\alpha}}{Q^{2}}\right) \\
= & \frac{q^{\alpha}}{Q^{2}} a^{n} 2 C_{n-2}^{(2)}(\eta)+p^{\alpha} a^{n-1} C_{n-1}^{(2)}(\eta),
\end{aligned}
$$

where we have used the following formulas

$$
\begin{gathered}
\frac{\partial a}{\partial q_{\alpha}}=a\left(\frac{-q^{\alpha}}{Q^{2}}\right), \quad \frac{\partial \eta}{\partial q_{\alpha}}=\frac{p^{\alpha}}{2 a}+\eta \frac{q^{\alpha}}{Q^{2}}, \\
\frac{d C_{n}^{(\nu)}(\eta)}{d \eta}=2 \nu C_{n-1}^{(\nu+1)}(\eta),
\end{gathered}
$$

and, at the last line, the recursion relation (B6). Further, we differentiate the both sides of Eq. (C5) with respect to $q_{\beta}$. Again using the formulas in (C6) and the recursion relation (B6), we get 


$$
\begin{aligned}
\frac{\partial}{\partial q_{\alpha}} \frac{\partial}{\partial q_{\beta}}\left(a^{n} C_{n}^{(1)}(\eta)\right)= & \frac{g^{\alpha \beta}}{Q^{2}} a^{n} 2 C_{n-2}^{(2)}(\eta) \\
& +\frac{q^{\alpha} q^{\beta}}{Q^{4}} a^{n} 8 C_{n-4}^{(3)}(\eta) \\
& +p^{\alpha} p^{\beta} a^{n-2} 2 C_{n-2}^{(3)}(\eta) \\
& +\frac{p^{\alpha} q^{\beta}+q^{\alpha} p^{\beta}}{Q^{2}} a^{n-1} 4 C_{n-3}^{(3)}(\eta) .
\end{aligned}
$$

Thus, from Eqs. (C4) and (C7), we obtain

$$
\begin{aligned}
q_{\mu_{1}} \cdots & q_{\mu_{n-2}}\left\{p^{\alpha} p^{\beta} p^{\mu_{1}} \cdots p^{\left.\mu_{n-2}\right\}_{n}}\right. \\
= & \frac{1}{n(n-1)}\left[\frac{g^{\alpha \beta}}{Q^{2}} a^{n} 2 C_{n-2}^{(2)}(\eta)\right. \\
& +\frac{q^{\alpha} q^{\beta}}{Q^{4}} a^{n} 8 C_{n-4}^{(3)}(\eta)+p^{\alpha} p^{\beta} a^{n-2} 2 C_{n-2}^{(3)}(\eta) \\
& \left.+\frac{p^{\alpha} q^{\beta}+q^{\alpha} p^{\beta}}{Q^{2}} a^{n-1} 4 C_{n-3}^{(3)}(\eta)\right],
\end{aligned}
$$

which is Eq. (2.11).

\section{APPENDIX D: NACHTMANN MOMENTS OF $F_{L}^{\gamma}$}

By applying the orthogonality relation (B4) for $\nu=3$ to the longitudinal amplitude (3.14) with the help of (3.15) we derive the following recursive relation for $M_{L, n}^{\gamma}$ 's:

$$
M_{L, n}^{\gamma}-2 \kappa \frac{n+1}{n+4} M_{L, n+2}^{\gamma}+\kappa^{2} \frac{(n+1)(n+2)}{(n+4)(n+5)} M_{L, n+4}^{\gamma}=I_{n}
$$

where

$$
\begin{aligned}
I_{n}= & \int_{0}^{x_{\max }} \frac{d x}{x^{5}} \xi^{n+3}\left[\frac{3+3(n+3) r+(n+2)(n+4) r^{2}}{(n+4)(n+5)}\right] \\
& \times F_{L}^{\gamma}\left(x, Q^{2}, P^{2}\right)+4 \kappa \frac{1}{n+2} M_{2, n+2}^{\gamma} \\
& -4 \kappa^{2} \frac{(n+1)(n+2)}{(n+3)(n+4)^{2}} M_{2, n+4}^{\gamma} .
\end{aligned}
$$

The above recursive Eq. (D1) can be solved as an infinite series:

$$
M_{L, n}^{\gamma}=(n+1) \sum_{l=0}^{\infty} \kappa^{l} \frac{(l+1)(n+2+l)}{(n+2 l+1)(n+2 l+2)} I_{n+2 l} .
$$

Introducing a variable $t$ defined by $\xi(P / Q) \equiv t$ we have

$$
\begin{gathered}
\xi \sqrt{\kappa}=t, \quad r=\frac{1-t^{2}}{1+t^{2}}, \quad \kappa \xi x=\frac{t^{2}}{1+t^{2}}, \\
\int_{0}^{x_{\max }} d x[\cdots]=\int_{0}^{1} d \xi \frac{1-t^{2}}{\left(1+t^{2}\right)^{2}}[\cdots],
\end{gathered}
$$

and then in terms of $t$ we can sum up the above infinite series and find

$$
\begin{aligned}
M_{L, n}^{\gamma}= & \int_{0}^{1} d \xi \xi^{n} \frac{1-t^{2}}{1+t^{2}}\left[\frac{1}{x^{2}} F_{L}^{\gamma}\left(x, Q^{2}, P^{2}\right)\right. \\
& \left.+4 \kappa \frac{(n+3)-(n+1) t^{2}}{(n+2)(n+3)} F_{2}^{\gamma}\left(x, Q^{2}, P^{2}\right)\right],
\end{aligned}
$$

the right-hand side of which turns out to be the Nachtmann moments (3.3), $\mu_{L, n}^{\gamma}$.
[1] T. F. Walsh, Phys. Lett. 36B, 121 (1971); S. J. Brodsky, T. Kinoshita, and H. Terazawa, Phys. Rev. Lett. 27, 280 (1971).

[2] T. F. Walsh and P. M. Zerwas, Phys. Lett. 44B, 195 (1973); R. L. Kingsley, Nucl. Phys. 60, 45 (1973).

[3] N. Christ, B. Hasslacher, and A. H. Mueller, Phys. Rev. D 6, 3543 (1972).

[4] E. Witten, Nucl. Phys. B120, 189 (1977).

[5] W. A. Bardeen and A. J. Buras, Phys. Rev. D 20, 166 (1979); 21, 2041(E) (1980).

[6] R. J. DeWitt, L. M. Jones, J. D. Sullivan, D. E. Willen, and H. W. Wyld, Jr., Phys. Rev. D 19, 2046 (1979); 20, 1751(E) (1979).

[7] M. Glück and E. Reya, Phys. Rev. D 28, 2749 (1983).

[8] K. Sasaki, Phys. Rev. D 22, 2143 (1980); Prog. Theor. Phys. Suppl. 77, 197 (1983).

[9] M. Stratmann and W. Vogelsang, Phys. Lett. B 386, 370 (1996).

[10] M. Glück, E. Reya, and C. Sieg, Phys. Lett. B 503, 285 (2001); Eur. Phys. J. C 20, 271 (2001).
[11] T. Uematsu and T. F. Walsh, Phys. Lett. 101B, 263 (1981).

[12] T. Uematsu and T. F. Walsh, Nucl. Phys. B199, 93 (1982).

[13] G. Rossi, Phys. Rev. D 29, 852 (1984).

[14] M. Drees and R. M. Godbole, Phys. Rev. D 50, 3124 (1994).

[15] M. Glück, E. Reya, and M. Stratmann, Phys. Rev. D 51, 3220 (1995); 54, 5515 (1996).

[16] M. Fontannaz, Eur. Phys. J. C 38, 297 (2004).

[17] K. Sasaki and T. Uematsu, Phys. Rev. D 59, 114011 (1999).

[18] K. Sasaki and T. Uematsu, Phys. Lett. B 473, 309 (2000); Eur. Phys. J. C 20, 283 (2001).

[19] K. Sasaki, T. Ueda, and T. Uematsu, Phys. Rev. D 73, 094024 (2006).

[20] M. Krawczyk, in PHOTON 2000: International Conference on the Structure and Interactions of the Photon, edited by A. J. Finch, AIP Conf. Proc. No. 571 (AIP, New York, 2001) and references therein; M. Krawczyk, A. Zembrzuski, and M. Staszel, Phys. Rep. 345, 265 (2001); R. Nisius, Phys. Rep. 332, 165 (2000); 
M. Klasen, Rev. Mod. Phys. 74, 1221 (2002); I. Schienbein, Ann. Phys. (N.Y.) 301, 128 (2002); R. M. Godbole, Nucl. Phys. B, Proc. Suppl. 126, 414 (2004).

[21] T. Ueda, K. Sasaki, and T. Uematsu, Phys. Rev. D 75, 114009 (2007).

[22] S. Moch, J. A. M. Vermaseren, and A. Vogt, Nucl. Phys. B688, 101 (2004).

[23] A. Vogt, S. Moch, and J. A. M. Vermaseren, Nucl. Phys. B691, 129 (2004).

[24] A. Vogt, S. Moch, and J. A. M. Vermaseren, Acta Phys. Pol. B 37, 683 (2006); arXiv:hep-ph/0511112.

[25] O. Nachtmann, Nucl. Phys. B63, 237 (1973); B78, 455 (1974).

[26] H. Georgi and H. Politzer, Phys. Rev. D 14, 1829 (1976).

[27] A. De Rujula, H. Georgi, and H. Politzer, Ann. Phys. (N.Y.) 103, 315 (1977); Phys. Rev. D 15, 2495 (1977).

[28] S. Wandzura, Nucl. Phys. B122, 412 (1977).

[29] S. Matsuda and T. Uematsu, Nucl. Phys. B168, 181 (1980).

[30] H. Kawamura and T. Uematsu, Phys. Lett. B 343, 346 (1995).

[31] A. Piccione and G. Ridolfi, Nucl. Phys. B513, 301 (1998).

[32] J. Blümlein and A. Tkabladze, Nucl. Phys. B553, 427 (1999).
[33] H. Baba, K. Sasaki, and T. Uematsu, Phys. Rev. D 65, 114018 (2002).

[34] S. Simula, Phys. Lett. B 574, 189 (2003).

[35] F. M. Steffens and W. Melnitchouk, Phys. Rev. C 73, 055202 (2006).

[36] For a recent review, see, for example, I. Schienbein et al., arXiv:0709.1775.

[37] Ch. Berger et al. (PLUTO Collaboration), Phys. Lett. 142B, 119 (1984).

[38] M. Acciarri et al. (L3 Collaboration), Phys. Lett. B 483, 373 (2000).

[39] R. Nisius, Phys. Rep. 332, 165 (2000).

[40] M. Glück, E. Reya, and I. Schienbein, Phys. Rev. D 63, 074008 (2001).

[41] V. M. Budnev, V. L. Chernyak, and I. F. Ginzburg, Nucl. Phys. B34, 470 (1971).

[42] K. Sasaki, J. Soffer, and T. Uematsu, Phys. Rev. D 66, 034014 (2002).

[43] Ch. Berger and W. Wagner, Phys. Rep. 146, 1 (1987).

[44] A. Erdélyi et al., Higher Transcendental Functions, Bateman Manuscript Project (McGraw-Hill, New York, 1953), Vol. I.

[45] I. S. Gradshteyn and I. M. Ryzhik, Tables of Integrals, Series, and Products (Academic Press, New York, 1965). 